\title{
Soil Transmitted Helminth Infections Among Children Admitted to Hospital Tengku Ampuan Afzan, Kuantan, Pahang
}

\author{
Abdullah Asady ${ }^{1}$, Pakeer Oothuman ${ }^{1}$, Soraya Ismail ${ }^{1}$, Marsitah Abdul Jalil ${ }^{2}$ \\ ${ }^{1}$ Department of Basic Medical Science, Kulliyyah of Medicine, International Islamic \\ University Malaysia \\ ${ }^{2}$ Hospital Tengku Ampuan Afzan, Kuantan
}

Presenter: Abdullah Asady

Introduction: Soil-transmitted helminth (STH) infections by Ascaris lumbricoides, Trichuris trichiura and hookworms still persist in poor communities in rural and urban areas. Recent studies in Malaysia focused on Orang Asli communities and few in hospital settings. This study determines the prevalence and risk factors for STH among children admitted to Paediatric ward of Hospital Tengku Ampuan Afzan (HTAA). Materials and Methods: This study was conducted among 133 (77 males, 56 females) paediatric patients in Hospital Tengku Ampuan Afzan from Dec 2017 to May 2018. Faecal samples were examined using wet smear, Kato-Katz, Harada-Mori and sedimentation techniques. Demographic data and hygiene practice information were collected using a pre-tested questionnaire. Results: The overall prevalence of STHs was $6 \%$ (A.lumbricoides $5.3 \%$, T.trichiura $0.8 \%$, and hookworms $0.8 \%$ ). Prevalence among males was $3.9 \%$ and females $8.9 \%$. Majority $(94.7 \%)$ were Malays. There were two Orang Asli children and both were infected, one of whom had a mixed infection. Chi square analysis showed that the important risk factors for STH infections are education level of mother $(p<0.05)$ and father $(p<0.05)$, the source of drinking water $(p<0.05)$, and the method of trash disposal $(p<0.05)$. Conclusion: This study shows that the prevalence of STH infections is low in children due to better socio-economy and good hygiene practice. Differences in individual lifestyles and humid weather condition are probable reasons for sporadic infection to still exist. 